\title{
Gallstone Ileus: Clinical Presentation of a Benign Mechanical Intestinal Obstruction
}

\author{
Eduardo E. Montalvo-Jave Eduardo Alegre-Tamez \\ César Athie-Gutiérrez \\ Department of Surgery, Faculty of Medicine, National Autonomous University of \\ Mexico (UNAM) and 'Hospital General de México, OD', Mexico City, Mexico
}

\section{Key Words}

Gallstone · Ileus · Surgery · Obstruction · Cholelithiasis

\begin{abstract}
Gallstone ileus is a rare cause of small bowel obstruction which usually presents in elderly female patients and which has been associated with high morbidity and mortality rates. We present the case of a 63-year-old man who presented at our institution with symptoms of bowel obstruction. Abdominal X-ray and exploratory laparotomy revealed a large gallstone in the terminal ileus.
\end{abstract}

\section{Background}

Gallstone ileus is a rare complication of cholelithiasis and an uncommon cause of mechanical bowel obstruction $[1,2]$. However, there are areas where the incidence of gallstone ileus may be higher, such as Chile, Mexico and another nations [3-5]. The incidence of gallstone ileus in Mexico is similar to that in other reports, around 30-35 patients per 1,000,000 hospital admissions over a 30- and 45-year period, respectively [4, $6]$.

Gallstone ileus is characterized by occlusion of the intestinal lumen as a result of one or more gallstones. This condition is rare and, consequently, the diagnosis is frequently missed or unduly delayed [7], as occurred in the case reported herein. Gallstone obstruction is responsible for $1-4 \%$ of all intestinal obstructions and carries a significant complication rate $[2,8]$; mortality ranges between 12 and 27\% [8, 9]. Gallstone ileus occurs mainly in elderly patients, who often have serious concomitant diseases. The most 
common concomitant diseases are cardiovascular, pulmonary, and diabetes mellitus [8]. Frequently, gallstone ileus originates from a cholecystoduodenal fistula $(90 \%)[5,10]$.

In most cases, the symptoms of gallstone ileus are initially vague and intermittent, probably because the calculus causes only partial obstruction of the intestinal lumen as it passes down the digestive tract. Later, when the stone becomes firmly impacted and complete obstruction occurs, vomiting and abdominal distention ensues. Advanced dehydration and electrolyte imbalance are usually present at the time medical advice is sought. In all cases of gallstone ileus, classical findings through plain abdominal roentgenograms include intestinal obstruction or aberrant located gallstones and pneumobilia $[8,11]$; CT findings in gallstone ileus may result in faster diagnosis and treatment $[12,13]$.

Surgical treatment can either be open or laparoscopic; with the new endoscopic techniques the surgical procedure has been advocated because of its lower morbidity and mortality [14]. Recently, a interesting choice to treat some specific cases with gallstone ileus was described using extracorporal shock wave lithotripsy and argon plasma coagulation [15].

\section{Case Report}

A 63-year-old man presented to our institution with a history of abdominal pain, vomiting, and constipation for three weeks. The patient had a medical history significant for uncontrolled diabetes mellitus and coronary artery disease. Physical examination was significant for massive abdominal distention without signs of peritoneal irritation, fever of $38.4^{\circ} \mathrm{C}$, and the patient was not clinically jaundiced. Laboratory examination revealed an elevated white blood cell count $15.8 \times 10^{9} / \mathrm{l}$, severe dehydration and electrolyte imbalance (hyponatremia and prerenal azotemia). Plain abdominal X-rays were consistent with small bowel obstruction, and a radiopaque gallstone was seen in the pelvis. Pneumobilia was not seen in this patient (fig. 1).

After appropriate fluid resuscitation, the patient's hemodynamic condition improved and he was taken to the operating room for exploratory laparotomy. Surgical exploration revealed massively dilated loops of small bowel proximal to the distal ileum, without evidence of abscess or purulent fluid collections. A point of obstruction was seen $53 \mathrm{~cm}$ from the terminal ileum where an enterotomy was made revealing a large gallstone $(5 \times 4 \times 4 \mathrm{~cm})$. The gallstone was removed and the enterotomy repaired in two layers (ig. 2 , fig. 3 ). We then performed a completion cholecystectomy; the gallbladder contained multiple stones ranging from 5 to $10 \mathrm{~mm}$. It was adherent to the duodenum, and after tedious dissection a fistula between the gallbladder and the first portion of the duodenum was identified. The fistula was taken down and the duodenum repaired primarily. The patient had an uneventful postoperative course and was dismissed home on postoperative day six.

\section{Conclusion}

We conclude that the prognosis is better with an early diagnosis based on the suspicion of this entity and knowledge regarding this pathology. In general the clinical and radiological findings, such as plain abdominal X-rays, ultrasound and CT scan, provide enough data to support the diagnosis and treatment. While conservative management with nasogastric tube decompression and intravenous fluid may be attempted, impacted gallstones are not likely to pass, and most patients will require an operation. 
Fig. 1. Supine X-ray of the abdomen showing a large gallstone.

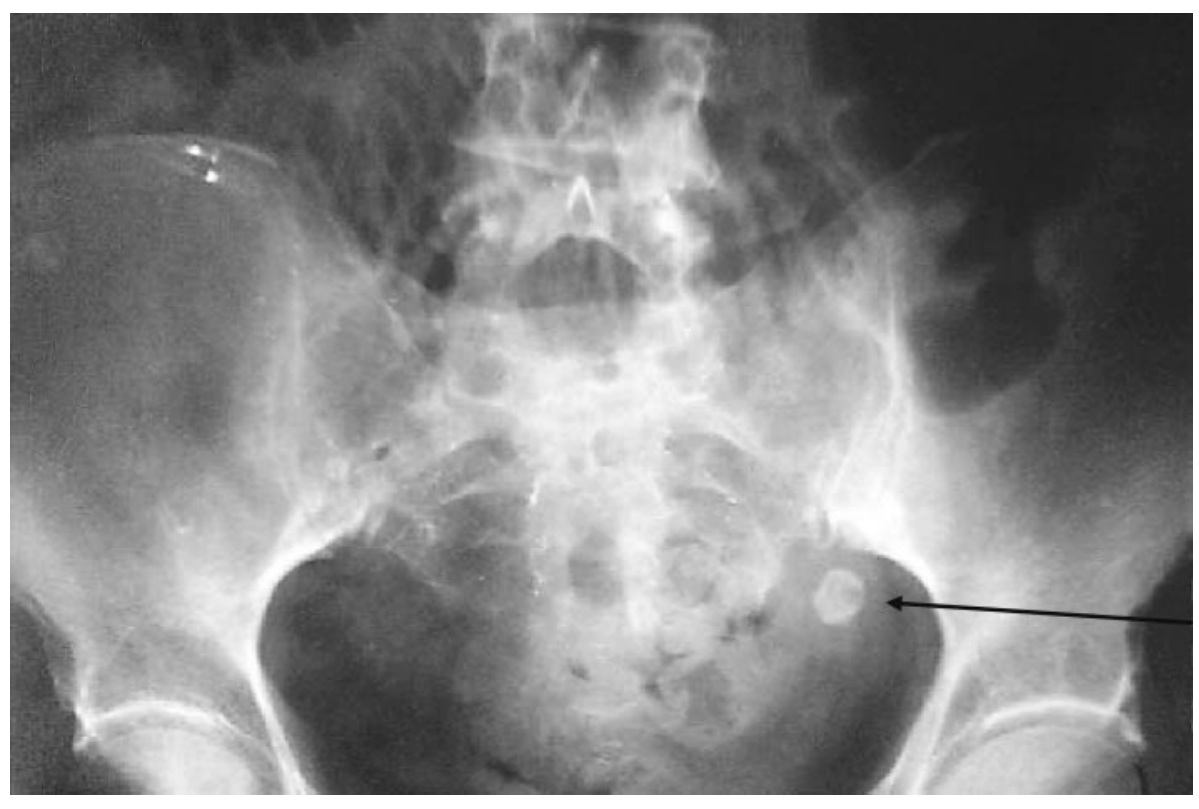

Fig. 2. Enterotomy.

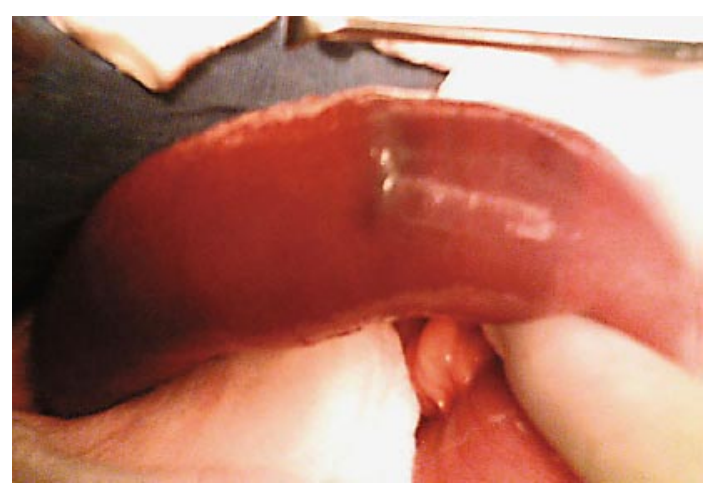


Fig. 3. Gallstone.

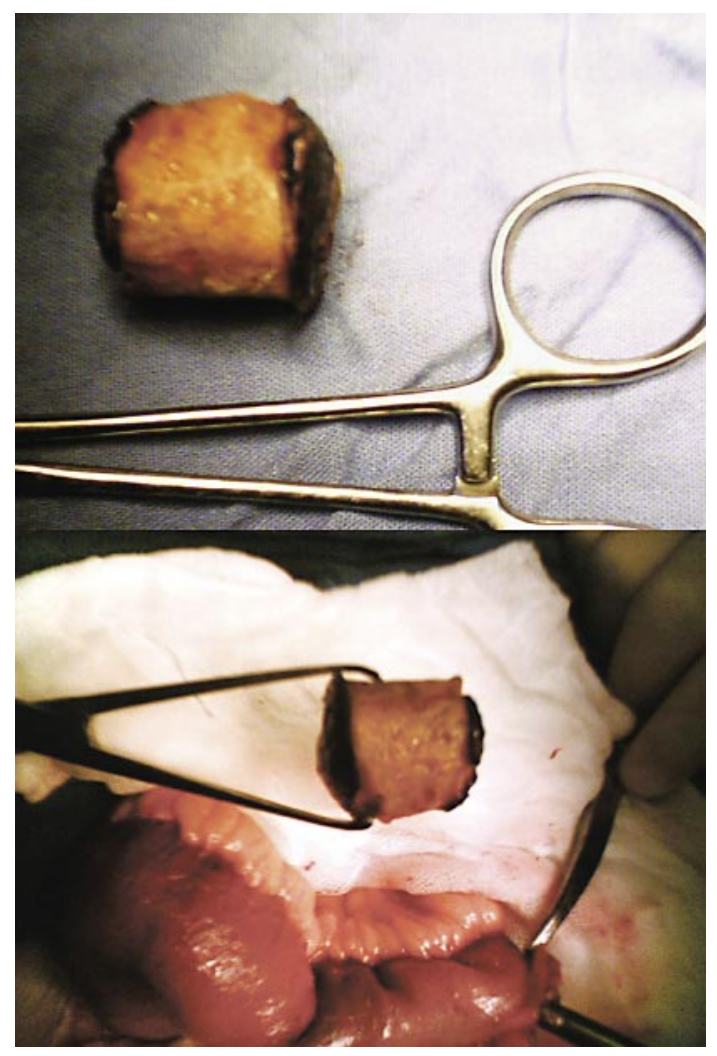




\section{References}

1 Deckoff SL: Gallstone ileus; a report of 12 cases. Ann Surg 1955;142:52-65.

2 Ayantunde AA, Agrawal EA: Gallstone ileus: diagnosis and management. World J Surg 2007;31:1292-1297.

-3 Schutte H, Bastías J, Csendes A, Yarmuch J, De la Cuadra R, Chiong H, Braghetto I: Gallstone ileus. Hepatogastroenterology 1992;39:562-565.

4 Masannat Y, Masannat Y, Shatnawei A: Gallstone ileus: a review. Mt Sinai J Med 2006;73:1132-1134.

5 González RV, Marenco CCA, Chávez GA, González DS, Montalvo JE: Laparoscopic cholecystectomy: results of the experience at Hospital General de México after nine years of implemented. Rev Mex Cir Endoscop 2002;3:71-73.

6 Kurtz RJ, Heimann TM, Beck AR, Kurtz AB: Pattern of treatment of gallstone ileus over a 45-year period. Am J Gastroenterol 1985;80:95-98.

7 Clavien PA, Richon J, Burgan S, Rohner A: Gallstone ileus. Br J Surg 1990;77:737742.

8 Reisner RM, Cohen JR: Gallstone ileus: a review of 1001 reported cases. Am Surg 1994;60:441-446.

-9 van Hillo M, van der Vliet JA, Wiggers T, Obertop H, Terpstra OT, Greep JM: Gallstone obstruction of the intestine: an analysis of ten patients and a review of the literature. Surgery 1987;101:273-276.

10 Abou-Saif A, Al-Kawas FH: Complications of gallstone disease: Mirizzi syndrome, cholecystocholedochal fistula, and gallstone ileus. Am J Gastroenterol 2002;97:249-254

11 Kurtz RJ, Heimann TM, Kurtz AB: Gallstone ileus: a diagnostic problem. Am J Surg 1983;146:314-317.

-12 Swift SE, Spencer JA: Gallstone ileus: CT findings. Clin Radiol 1998;53:451-454.

13 Graham JS, Rothwell BC: Images in clinical medicine. Gallstone ileus. N Engl J Med 2004;351:1119.

14 Moberg AC, Montgomery A: Laparoscopically assisted or open enterolithotomy for gallstone ileus. Br J Surg 2007;94:53-57.

15 Gemmel C, Weickert U, Eickhoff A, Schilling D, Riemann JF: Successful treatment of gallstone ileus (Bouveret's syndrome) by using extracorporeal shock wave lithotripsy and argon plasma coagulation. Gastrointest Endosc 2007;65:173175 . 